\title{
Desarrollo y validación de un método para cuantificación de acetaminofén en supositorios mediante HPLC-DAD utilizando una variación del método QuEChERS
}

Arnulfo Rodríguez y Yeffry Martínez ${ }^{1}$

Henry Ponce ${ }^{2}$

\section{RESUMEN}

Se propone un método para la identificación y cuantificación de acetaminofén en supositorios mediante cromatografía líquida de alta resolución con detector de arreglo de diodos (HPLC-DAD), utilizando una variación del método de extracción QuEChERS. Se empleó la separación cromatográfica utilizada en el método de la farmacopea de los Estados Unidos, edición 35 de 2012, en la que se practica un gradiente isocrático, con una fase móvil compuesta de agua y metanol (70:30). La columna utilizada fue C18 (L1) de $150 \times 4.6 \mathrm{~mm}$, con un tamaño de partícula de $5 \mu \mathrm{m}$, manteniendo la columna a una temperatura de $45^{\circ} \mathrm{C}$ y el detector se colocó a una longitud de onda de $243 \mathrm{~nm}$. Para el proceso de extracción y purificación del analito se utilizó una técnica diferente al que señala la farmacopea, en donde se aplica una extracción líquido-líquido, obteniéndose con el procedimiento desarrollado, elevados valores de recuperación del analito. Los resultados de la validación del método mostraron valores que cumplieron los criterios de aceptación de los parámetros evaluados. El método desarrollado puede ser aplicado para el análisis de acetaminofén en supositorios, con iguales resultados al de la farmacopea, con la ventaja de ser más rápido, menos costoso y amigable con el ambiente.

Palabras claves: QuEChERS, HPLC, validación, supositorios, farmacopea.

1 Estudiantes, Departamento de Control Químico, Facultad de Química y Farmacia, UNAH: aerm092@gmail.com, yefrinahunm@gmail.com

${ }^{2}$ Asesor, Departamento de Control Químico, Facultad de Química y Farmacia, UNAH: henry.ponce@unah.edu.hn 


\section{ABSTRACT}

A method for the identification and quantification of acetaminophen suppositories is proposed by high performance liquid chromatography with diode array detector (HPLC-DAD), using a variation of QuEChERS extraction method. Chromatographic separation used in the method of US Pharmacopoeia, 35 edition of 2012, in which an isocratic gradient practiced with a mobile phase composed of water and methanol (70:30) was used. The column used was C18 (L1), $150 \times 4.6 \mathrm{~mm}$, with a particle size of $5 \mu \mathrm{m}$, maintaining the column at a temperature of $45^{\circ} \mathrm{C}$, and the detector was placed at a wavelength of $243 \mathrm{~nm}$. For the process of extraction and purification of the analyte to a different technique pointing pharmacopoeia it was used, wherein a liquid-liquid extraction, obtained with the procedure developed high values of analyte recovery. The results of method validation process showed values that met the acceptance criteria of the parameters evaluated. The method developed can be applied to the analysis of acetaminophen suppositories, with similar results to the pharmacopoeia, with the advantage of being faster, less expensive and environmentally friendly.

Keywords: QuEChERS, HPLC, validation, suppositories, pharmacopoeia. 


\section{INTRODUCCIÓN}

El fármaco acetaminofén es el metabolito activo de la fenacetina, siendo utilizado en lugar de la aspirina como analgésico-antipirético, con poca actividad antinflamatoria (Roberts II y Morrow, 2001). En nuestro país es utilizado ampliamente, encontrándose dentro del cuadro básico de medicamentos de los centros asistenciales públicos, administrándose en diferentes formas farmacéuticas.

El acetaminofén es administrado por vía rectal, como supositorio, para tratar muchas condiciones como dolor de cabeza, dolores musculares, artritis, dolor de espalda, dolores de muelas, resfriados, y fiebre. Su efecto antipirético se debe a que puede inhibir la ciclooxigensa en el encéfalo (Roberts II y Morrow, 2001). El acetaminofén presenta en su estructura química grupos funcionales importantes, dentro de los cuales el carbonilo le brinda la posibilidad de absorber radiación en la región ultravioleta (UV) del espectro electromagnético, con longitudes de onda máximas entre los 240 y $260 \mathrm{~nm}$, como se puede observar en la figura 1.

Figura 1. Estructura molecular y espectro de absorción UV del acetaminofén

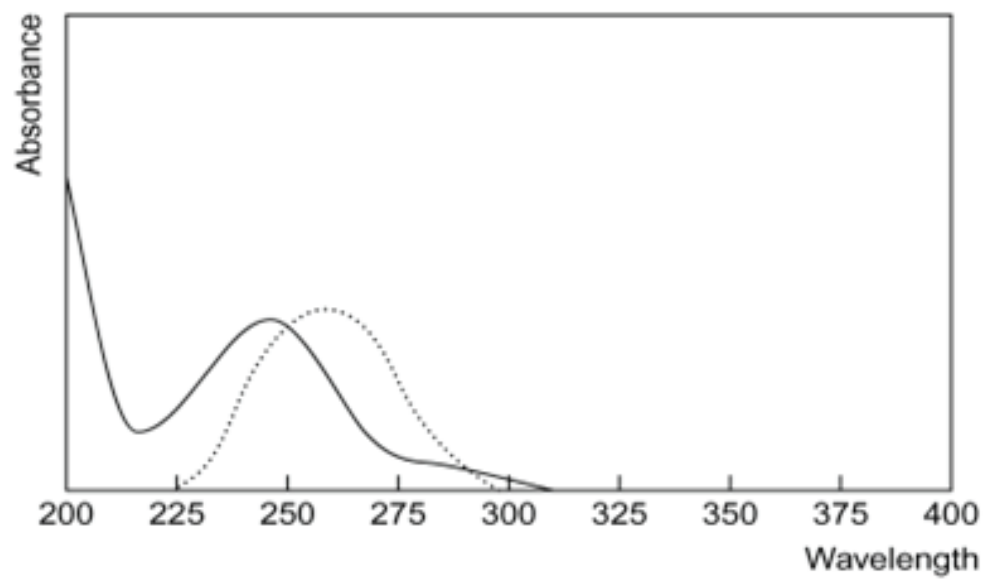

Fuente: Moffat, Osselton y Widdop, 2005.

En nuestro país, gran parte de los laboratorios farmacéuticos y de control de calidad de medicamentos utilizan como métodos de análisis los que contemplan los libros oficiales, entre estos los de la farmacopea de los Estados Unidos (USP, por sus siglas en inglés), que son los más utilizados. En ese sentido, para la valoración del acetaminofén en supositorios, la USP XXXV (Convención de la Farmacopea de los Estados Unidos de América, 2012) emplea la cromatografía líquida de elevada resolución 
(HPLC), con un modo de separación en fase reversa y establece como procedimiento para la preparación de la muestra una extracción líquido-líquido, con treinta mililitros de éter de petróleo, efectuando múltiples lavados con agua destilada.

En este contexto, los métodos por HPLC han demostrado ser la metodología analítica más utilizada para el control de calidad de medicamentos, siendo aplicados con mayor regularidad en nuestro país, sin que esto signifique que sea un método barato y al alcance todos los laboratorios. La cromatografía es una técnica física de separación de compuestos presentes en una mezcla, en la cual los componentes son distribuidos entre dos fases: una estacionaria y una móvil que se mueve en una dirección definida. En el caso de HPLC, la fase móvil es un líquido y la fase estacionaria puede tener características polares o apolares, pudiendo ser de diversos materiales, tales como silica, silica modificada, polímeros, entre otros (Snyder, Kirkland y Dolan, 2010).

Dentro del proceso analítico, una etapa fundamental para alcanzar los resultados deseados, es la preparación de la muestra, puesto que esta debe garantizar la separación, purificación y concentración del o los analitos de interés del resto de los componentes de la matriz. Pese a los grandes avances en la instrumentación analíti$\mathrm{ca}$, muchas normativas internacionales emplean técnicas de tratamiento de muestra tradicionales dentro de sus procedimientos analíticos, como en el caso de la referida USP XXXV para la valoración de acetaminofén en supositorios, aplicando la extracción líquido-líquido.

Es sabido que esta extracción presenta algunas desventajas importantes, ya que es laboriosa y con elevado consumo de solventes, los cuales en general presentan elevada toxicidad, tanto para el analista, como para el ambiente. Asimismo, en la extracción líquido-líquido no es posible la automatización del procedimiento, sobre todo cuando se cuenta con un elevado número de muestras a analizar (Mitra, 2003). Debido a estos inconvenientes han aparecido novedosas técnicas para el tratamiento de muestras, las cuales buscan convertirse en una técnica ideal de tratamiento de muestras, es decir, rápida, exacta, precisa, que garantice la integridad de la muestra y alto desempeño (Núñez, Gallart, Martins y Lucci, 2012).

Dentro de estas técnicas novedosas, la extracción QuEChERS (por sus siglas en inglés: quick, easy, cheap, effective, rugged and safe) ha venido ganando popularidad por su simplicidad, rapidez y aplicabilidad. Introducida por Anastassiades y colaboradores (2003), QuEChERS surge como un método de tratamiento de muestras de 
frutas y vegetales en la determinación de multirresiduos de pesticidas, basándose el método original en una extracción a micro escala utilizando acetonitrilo como solvente extractor, la extracción de los analitos contenidos en las muestras con alto contenido acuoso y una partición líquido-líquido utilizando sulfato de magnesio y cloruro de sodio; por último, una etapa opcional de limpieza de extracción en fase sólida dispersiva (d-SPE), utilizando como sorbente amina primaria secundaria (PSA) (Zhang y otros, 2012).

QuEChERS engloba dos etapas principales, comenzando por la etapa de extracción de fase simple, en la que se realiza la adición de un solvente miscible en agua, de carácter hidrófilo, utilizando normalmente acetonitrilo o acetona, a la porción de una muestra homogenizada previamente; esto permite la extracción de los analitos en el solvente. Pese a que ambos solventes son miscibles en agua, pueden separarse fácilmente de la fase acuosa utilizando la adición de una mezcla de sulfato de magnesio anhidro más cloruro de sodio, lo que a su vez promueve la extracción de los analitos en el solvente, por efecto del "salting-out".

La segunda etapa comprende una d-SPE opcional, que involucra la adición de una cantidad de sorbente en bruto, siendo las más utilizadas la amina primaria secundaria (PSA), carbón negro grafitizado (GCB), octadecilsilano (C18), silicagel, lo mismo que combinaciones de estas, para realizar una limpieza que remueva de forma efectiva muchos de los componentes polares presentes en la matriz, como ácidos orgánicos, lípidos, algunos pigmentos polares y azúcares, que permanecen en el extracto (Anastassiades, 2003). Actualmente existen tres variantes del método QuEChERS, los cuales se diferencian en función de los reactivos utilizados y se presentan a continuación:

1. Método original, introducido en 2003 , utiliza cloruro de sodio para mejorar la extracción.

2. Método dispersivo AOAC 2007.01. Se utiliza acetato de sodio como tampón en substitución del cloruro de sodio.

3. Método europeo EN15662. Similar al método AOAC utilizando cloruro de sodio, citrato de sodio dihidrato y citrato de sodio sequihidrato, en lugar del acetato de sodio. La figura 2 muestra el proceso QuEChERS con sus diferentes variantes. 
Figura 2. Esquema de las variantes de QuEChERS

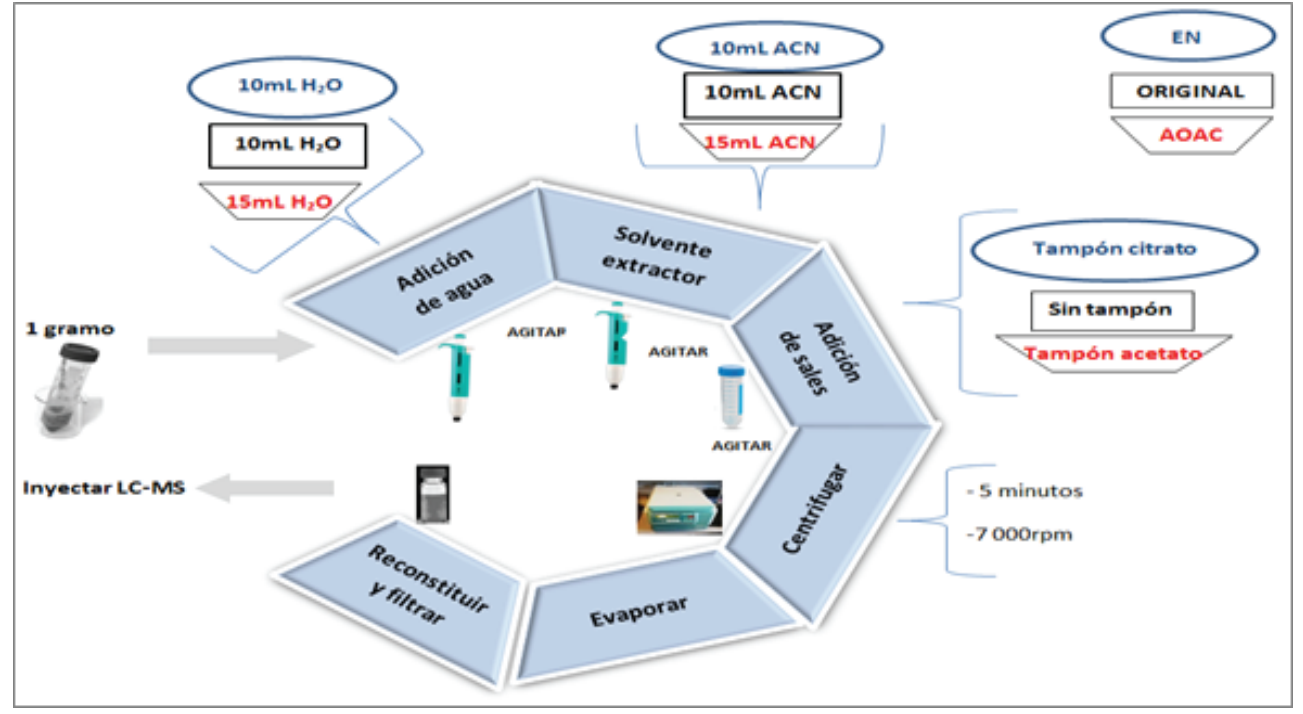

Fuente: Ponce, 2014.

Debido a las enormes ventajas y resultados que pueden obtenerse, la extracción QuEChERS ha sido aplicada en diversos campos de análisis, no solamente para el análisis de pesticidas en alimentos, donde fue originalmente aplicado, sino en el campo de contaminantes ambientales, análisis de alimentos y campo forense para el análisis de drogas de abuso, como se muestra en la tabla 1.

Uno de los aspectos más importante del desarrollo de métodos analíticos es la posterior validación de los mismos, proceso bajo el cual se demuestra, mediante experimentos y cálculos estadísticos, que el método funciona para el objeto que fue creado, en nuestro caso, para la identificación y cuantificación de acetaminofén en supositorios. Para tal fin, existen diferentes recomendaciones de validación de métodos, entre las cuales las más importantes son las de la Comisión Internacional de Armonización (ICH), la cual establece los principales parámetros de desempeño a evaluar, lo mismo que los criterios que deben evaluarse (Guideline, 2006); a nivel regional, el Reglamento Técnico Centroamericano es el organismo que dicta las guías para la validación de métodos analíticos para el análisis y control de medicamentos (RTCA, 2010). 


\section{Tabla 1. Resumen de aplicaciones de QuEChERS}

\begin{tabular}{|c|c|c|c|}
\hline Compuestos & Matriz & Campo de aplicación & $\begin{array}{c}\text { Método de separación } \\
\text { y detección }\end{array}$ \\
\hline $\begin{array}{c}\text { Hidrocarburos } \\
\text { aromáticos } \\
\text { policiclicos (PAHs) }\end{array}$ & Camarones & $\begin{array}{l}\text { Seguridad de } \\
\text { alimentos }\end{array}$ & (RP)LC-MS-MS \\
\hline $\begin{array}{c}\text { Hidrocarburos } \\
\text { aromáticos } \\
\text { policiclicos (PAHs) }\end{array}$ & Peces & $\begin{array}{l}\text { Seguridad de } \\
\text { alimentos }\end{array}$ & (RP)LC-fluorescencia \\
\hline Micotoxinas & Cereales & $\begin{array}{l}\text { Seguridad de } \\
\text { alimentos }\end{array}$ & (RP)LC-MSHR \\
\hline $\begin{array}{l}\text { Altihelmínticos } \\
\text { (albendazol) }\end{array}$ & Leche & $\begin{array}{l}\text { Seguridad de } \\
\text { alimentos }\end{array}$ & (RP)LC-MS \\
\hline Acrilamida & $\begin{array}{l}\text { Chocolate, patatas } \\
\text { fritas, frutas y } \\
\text { mantequilla de maní }\end{array}$ & $\begin{array}{l}\text { Seguridad de } \\
\text { alimentos }\end{array}$ & (RP)LC-MS-MS \\
\hline $\begin{array}{l}\text { Antibióticos y drogas } \\
\text { veterinarias }\end{array}$ & Tejido animal & Análisis biológicos & (RP)LC-MS-MS \\
\hline$\beta$ agonistas & $\begin{array}{c}\text { Suplementos } \\
\text { dietéticos (tabletas } \\
\text { y cápsulas) }\end{array}$ & $\begin{array}{l}\text { Seguridad de } \\
\text { medicamentos }\end{array}$ & (RP)LC-MSHR \\
\hline $\begin{array}{c}\text { Trialometanos, } \\
\text { benceno, tolueno, } \\
\text { etilbenceno y xileno }\end{array}$ & Suelo & Análisis ambientales & GC-MS \\
\hline Clorinados & Suelo & Análisis ambientales & GC-ECD \\
\hline $\begin{array}{c}\text { Benzotriazol, } \\
\text { benzotiazol y otros }\end{array}$ & $\begin{array}{l}\text { Lodos de } \\
\text { depuradora }\end{array}$ & Análisis ambientales & (RP)LC-MSHR \\
\hline $\begin{array}{c}\text { Pesticidas } \\
\text { organofosforados }\end{array}$ & Contenido gástrico & Análisis forense & GC-MS \\
\hline Drogas de abuso & Sangre & Análisis forense & (RP)LC-MS-MS \\
\hline
\end{tabular}

$\mathrm{RP}=$ Fase reversa; $\mathrm{LC}=$ Cromatografía líquida; $\mathrm{MS}=$ Espectrometría de masas; $\mathrm{GC}=$ Cromatografía de gases; $E C D=D e t e c t o r$ de captura de electrones; HR= Elevada resolución

Fuente: Ponce, 2014. 
El método de QuEChERS ha sido aplicado en diversos campos del análisis, pero hasta donde conocen los autores, no se han presentado trabajos de investigación que apliquen esta técnica de tratamiento de muestra para el análisis de medicamentos y precisamente supositorios, como alternativa a la extracción líquido-líquido que recomienda el método de la farmacopea. Es por ello que con el presente trabajo se buscó desarrollar un método alterno para determinar acetaminofén en supositorios, efectuando una variación al procedimiento de QuEChERS y empleando la separación mediante HPLC-DAD que recomienda la farmacopea. Las ventajas que se buscan con este método es que sea rápido, menos costoso, con elevadas recuperaciones de analito. Posteriormente, se efectuó la validación del método analítico siguiendo la guía de la ICH para la referida forma farmacéutica.

\section{MÉTODO}

\section{Diseño}

El método de investigación se rige por un diseño aleatorio, ya que no se controlaron las cantidades de acetaminofén en los supositorios, de modo que se trabajó con los supositorios comerciales adquiridos de diferentes establecimientos farmacéuticos. Asimismo, se realizó una revisión bibliográfica sobre el método de análisis para dicho compuesto en la forma farmacéutica de supositorios.

\section{Población y muestra}

Lotes de supositorios de acetaminofén de $300 \mathrm{mg}$ fueron proveídos de diferentes farmacias de consumo poblacional. Se utilizó un estándar grado USP obtenido de los laboratorios docentes de la Facultad de Ciencias Químicas y Farmacia de la Universidad Nacional Autónoma de Honduras para llevar a cabo todos los experimentos.

\section{Entorno}

El estudio se efectuó en la Facultad de Ciencias Químicas y Farmacia en el laboratorio instrumental, en donde se encuentran el cromatógrafo líquido, baño ultrasónico, agitador mecánico, centrifuga, micropipetas y el resto de equipamiento de laboratorio. 


\section{Intervenciones}

Para el desarrollo del método se utilizó un baño ultrasónico, marca Fisher y un agitador mecánico de la marca KoolLab, modelo KS-VM-1000, centrifuga marca Clay Adams. La separación cromatográfica se efectuó en un cromatógrafo líquido marca Shimadzu, modelo Prominence, consistiendo en una bomba modelo LC20-AT, un sistema de control CMB-20Alite, un inyector automático SIL-20A, un horno de columna CTO-A20 y un detector SPD-M20A. Se utilizó el software EZ Start para recolectar todos los datos obtenidos durante el proceso de investigación.

En el desarrollo de las condiciones cromatográficas se utilizó una columna Alltima C18 de $150 \mathrm{~mm}$ de longitud y $4.6 \mathrm{~mm}$ de diámetro interno, con un tamaño de partícula de $5 \mu \mathrm{m}$, a una temperatura del horno de $45^{\circ} \mathrm{C}$. El volumen de flujo de la fase móvil fue de $1,5 \mathrm{~mL} / \mathrm{min}$ y el volumen de inyección se fijó en $15 \mu \mathrm{L}$ de las soluciones del estándar y las muestras. El detector de arreglo de diodos se controló a una longitud de onda de $243 \mathrm{~nm}$. La fase móvil empleada consistió en una mezcla de agua-metanol (70:30). Acetonitrilo y metanol calidad HPLC fueron utilizados de la marca J.T. Baker y se adquirieron de la casa comercial Labtech, ubicada en Tegucigalpa, Honduras. Sulfato de magnesio, acetato de sodio, ácido acético glacial y silicagel, todos de la marca Merck, fueron adquiridos del almacén de reactivos de la Facultad de Ciencias Químicas y Farmacia.

\section{Análisis estadístico}

Para analizar todos los datos obtenidos en los experimentos del desarrollo y validación del método propuesto, se utilizaron hojas de cálculo de Microsoft Excel 2010, además del software del instrumento EZ Start versión 7.4. Debido a que en el proceso de validación debe definirse qué será evaluado y cómo, es decir, qué criterios de calidad del método serán examinados y bajo qué criterios será realizada dicha evaluación. Todo ello dependerá de una serie de factores relacionados con las características de la matriz, complejidad del método analítico propuesto, exactitud y precisión con que deban expresarse los resultados y la aplicación que tendrá el método propuesto, entre otros aspectos. Para ello, se siguieron las recomendaciones de la $\mathrm{ICH}$, las cuales aparecen en la tabla 2. 
Tabla 2. Parámetros y criterios recomendados por la ICH

\begin{tabular}{|c|c|}
\hline Parámetro & Criterio \\
\hline Especificidad & $\begin{array}{l}\text { Emplear un test de pureza de pico con un detector de arreglo de } \\
\text { diodos (DAD) }\end{array}$ \\
\hline Linealidad & $\begin{array}{l}\text { a. Evaluación del gráfico de calibración lineal } \\
\text { b. Ecuación de la recta } \\
\text { c. Coeficientes de correlación }(r) \geq 0.998 \text { y determinación }\left(r^{2}\right) \geq 0.99 \\
\text { d. Análisis de varianza (prueba } F \text { ) }\end{array}$ \\
\hline Rango de trabajo & $\begin{array}{l}\text { Obtenido del ítem anterior, donde se cumplan los criterios de } \\
\text { exactitud, precisión y linealidad }\end{array}$ \\
\hline $\begin{array}{l}\text { Exactitud } \\
\text { (veracidad) }\end{array}$ & $\begin{array}{l}\text { Porcentaje de recuperación en el ensayo de la cantidad de analito } \\
\text { reforzado, porcentaje de recuperación entre } 90 \%-110 \%\end{array}$ \\
\hline Repetibilidad & $\begin{array}{l}\text { Análisis de precisión en corto intervalo de tiempo: mismo día, } \\
\text { instrumento y analista, evaluación de RSD (\%CV); el criterio será } \\
\text { menor } 2 \% \text { para el \%CV }\end{array}$ \\
\hline $\begin{array}{l}\text { Reproducibilidad } \\
\text { intermedia }\end{array}$ & $\begin{array}{l}\text { a. Análisis de precisión en condiciones amplias de variación: días; } \\
\text { evaluación de RSD }(\% \mathrm{CV}) \text {, el criterio será menor } 2 \% \text { para el \%CV } \\
\text { b. Prueba t para la variación entre días }\end{array}$ \\
\hline
\end{tabular}

Fuente: Creación propia.

\section{RESULTADOS Y DISCUSIONES}

\section{Optimización de la separación cromatográfica}

Para la separación cromatográfica se emplearon las condiciones recomendadas por el método de la farmacopea, con las cuales se obtuvo el pico cromatográfico correspondiente al analito poco después de los dos minutos, como se muestra en la figura 3 , en donde aparece además del cromatograma de una solución estándar, el espectro de absorción ultravioleta característico del acetaminofén. 


\section{Optimización del procedimiento del método QuEChERS}

En la optimización de los diferentes parámetros del método QuEChERS se optó por trabajar con algunas condiciones iniciales, efectuando la disolución de la muestra de supositorios, previamente fundidos a baño a maría, en $10 \mathrm{ml}$ de agua destilada, para después agregar $10 \mathrm{ml}$ de dos posibles solventes extractores, acetona y acetonitrilo; asimismo, evaluando el uso de tampón de acetato frente a la extracción sin utilizar regulación de $\mathrm{pH}$.

Figura 3. Cromatograma obtenido con las condiciones finales del método

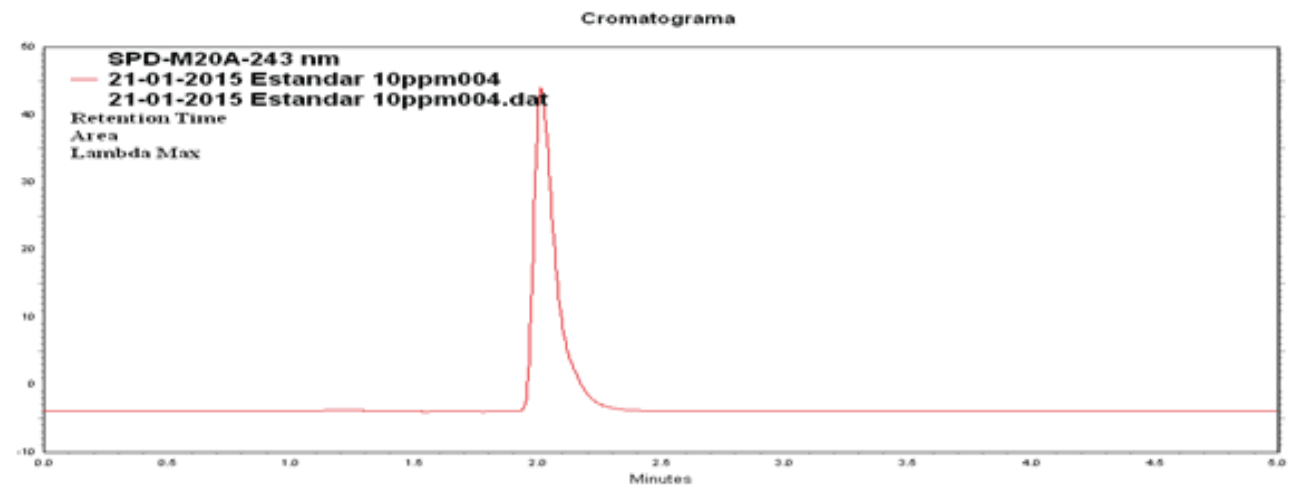

Fuente: Cromatógrafo marca Shimadzu

En el primero de los casos, se observaron mejores resultados en la recuperación del analito cuando se utilizó acetona sin regulación de $\mathrm{pH}$, pero los valores fueron más elevados al combinar la extracción con acetonitrilo y tampón de acetato y ácido acético, con una recuperación cercana al $50 \%$. Esto puede explicarse debido a que el valor de pKa del acetaminofén es de 9,5 (Moffat, Osselton y Widdop, 2005) y al emplear el tampón de acetato, el cual regula la extracción a un pH cercano a 4,8 estando más alejado del valor donde el analito puede encontrarse ionizado, lo que lo convertiría más a fin a la fase acuosa, disminuyendo de esta forma la recuperación en el acetonitrilo. Los porcentajes encontrados se pueden observar en el gráfico 1.

Luego de realizar diferentes experimentos para incrementar los porcentajes de recuperación, empleando diferentes cantidades de agua y acetonitrilo, lo mismo que variando la temperatura del solvente acuoso, se decidió emplear un solvente apolar, de modo que se pudiera separar el excipiente oleoso presente en el supositorio. La mejor opción a emplear fue el hexano, solvente de baja polaridad, el cual fue capaz 
de solubilizar completamente la fase oleosa, dejando liberado el acetaminofén para que este pasará al acetonitrilo. En este punto se repitieron las extracciones con acetona, para evaluar su uso como solvente extractor, pero debido a que presenta miscibilidad en hexano se descartó como solvente extractor. Finalmente, se confirmó el uso de tampón con hexano y acetonitrilo, alcanzando una recuperación mayor al 80 $\%$, resultados mostrados en el gráfico 2 .

\section{Gráfico 1. Evaluación del solvente extractor y uso de tampón}

\section{Evaluación del disolvente extractor y uso de tampón}

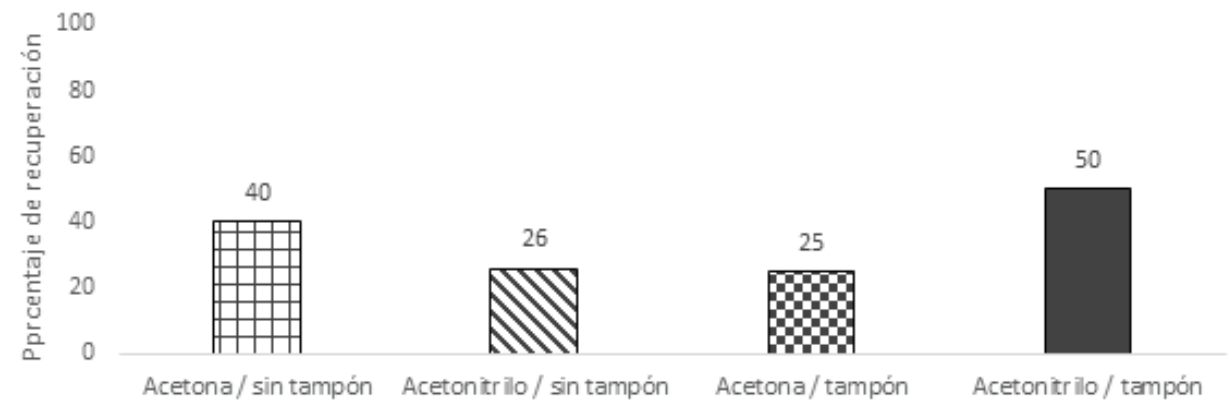

Condiciones evaluadas

Fuente: Creación propia.

\section{Gráfico 2. Optimización del solvente de la muestra}

\section{Evaluación del disolvente de la muestra y tampón}
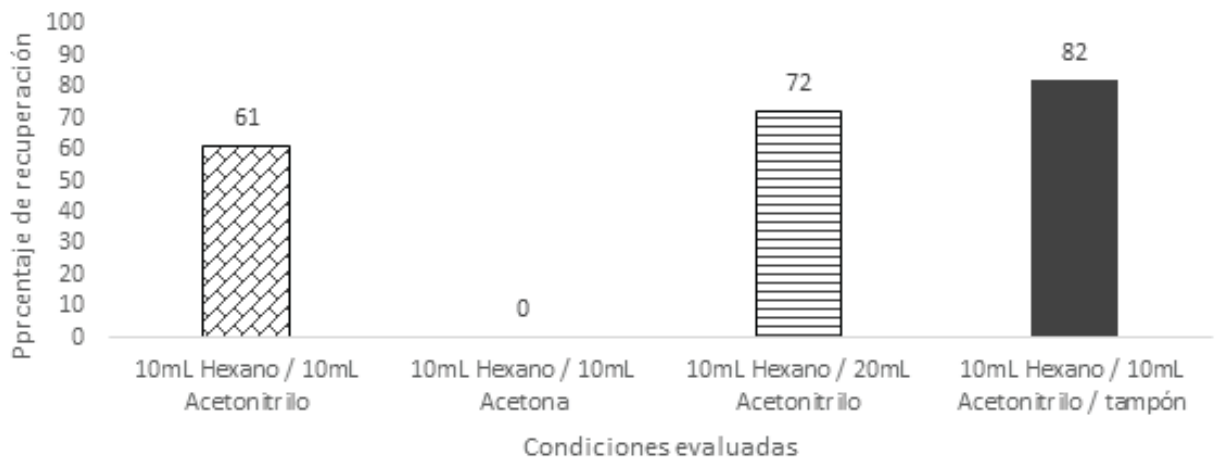

Fuente: Creación propia.

Para finalizar la optimización de la primera etapa de la extracción y en búsqueda de obtener un método más barato, se efectuaron pruebas para disminuir el uso de solventes y sales, lo mismo que el tiempo de centrifugación. Por ello se practicaron cinco extracciones, modificando las condiciones que se observan en la tabla 3. 


\section{Tabla 3. Optimización de primera etapa de QuEChERS}

\begin{tabular}{|l|c|}
\hline Condiciones evaluadas & Porcentaje recuperado \\
\hline $5 \mathrm{ml}$ de hexano; $10 \mathrm{ml}$ de acetonitrilo; $6 \mathrm{~g} \mathrm{MgSO}_{4} ; 1,5 \mathrm{~g} \mathrm{CH}_{3} \mathrm{COONa}$ & $68 \%$ \\
\hline $10 \mathrm{ml}$ de hexano; $20 \mathrm{ml}$ de acetonitrilo; $6 \mathrm{~g} \mathrm{MgSO}_{4} ; 1,5 \mathrm{~g} \mathrm{CH}_{3} \mathrm{COONa}$ & $87 \%$ \\
\hline $\begin{array}{l}5 \mathrm{ml} \text { de hexano; } 10 \mathrm{ml} \text { de acetonitrilo; } 3 \mathrm{~g} \mathrm{MgSO}_{4} ; 0,75 \mathrm{~g} \mathrm{CH}_{3} \mathrm{COONa} ; 5 \\
\text { min centrifugación }\end{array}$ & $96 \%$ \\
\hline $\begin{array}{l}5 \mathrm{ml} \text { de hexano; } 10 \mathrm{ml} \text { de acetonitrilo; } 1,5 \mathrm{~g} \mathrm{MgSO}_{4} ; 0,37 \mathrm{~g} \mathrm{CH}_{3} \mathrm{COONa} ; \\
5 \mathrm{~min} \text { centrifugación }\end{array}$ & \\
\hline $\begin{array}{l}10 \mathrm{ml} \text { de hexano; } 10 \mathrm{ml} \text { de acetonitrilo; } 1,5 \mathrm{~g} \mathrm{MgSO}_{4} ; 0,37 \mathrm{~g} \\
\mathrm{CH}_{3} \mathrm{COONa} ; 5 \text { min centrifugación }\end{array}$ & \\
\hline
\end{tabular}

Fuente: Creación propia.

El valor más alto de recuperación de acetaminofén fue cuando se emplearon 5 mililitros de hexano para solubilizar la muestra, más 10 mililitros de acetonitrilo con ácido acético al $1 \%$ y añadiendo $1,5 \mathrm{~g}$ de sulfato de magnesio más $0,37 \mathrm{~g}$ de acetato de sodio, empleando 5 minutos para centrifugar el extracto. Como puede verse, la variación del doble volumen de hexano no modifica el porcentaje de recuperación, pero al trabajar con menor cantidad de este solvente se vuelve más barato el método, lo mismo que menos producción de desechos químicos. Asimismo, la utilización de una menor cantidad de sales abarata los costos del análisis.

Con respecto a la optimización de la etapa de extracción en fase sólida dispersiva del método, la cual busca una purificación del extracto obtenido, se experimentó únicamente con silicagel como sorbente, ya que solo no se disponía de los otros sorbentes normalmente empleados en QuEChERS. Sin embargo, se realizaron pruebas para optimizar la cantidad a utilizar, lo mismo que el tiempo de centrifugación en esta etapa. Los resultados alcanzados se observan en el gráfico 3.

Pese a que el mayor porcentaje se encontró con un gramo de silicagel, la diferencia al utilizar la mitad de esa cantidad no es significativamente diferente, además de que puede existir una variación mayor a la diferencia de ambas pruebas, por lo que se decidió trabajar con la cantidad de $0,5 \mathrm{~g}$ de silicagel y un tiempo igual a 5 minutos de centrifugación. Con estos resultados, en la figura 4 se muestra el procedimiento esquematizado de la extracción optimizada. 


\section{Validación del método}

Siguiendo las recomendaciones de la $\mathrm{ICH}$, se procedió a validar los distintos parámetros que aparecen en la tabla 2, excluyendo el límite de detección y límite de cuantificación, debido a que no son necesarios cuando el método que se utiliza lleva como finalidad la valoración del analito presente en los supositorios.

1. Especificidad: aprovechando que el detector con que cuenta el instrumento es uno de arreglo de diodos (DAD) y ya que se presentó la imposibilidad de contar con material de referencia de los productos de degradación y excipientes del preparado farmacéutico, se decidió evaluar la especificidad a través de la pureza de pico cromatográfico, comparando el espectro de absorción del acetaminofén de la figura 3 , con el espectro de absorción teórico, con lo que se demostró que el pico corresponde al del analito de interés.

2. Linealidad y rango de trabajo: para la evaluación de la linealidad se preparó una curva de calibración por triplicado en días diferentes con concentraciones finales desde $1.0 ; 2.5 ; 5.0 ; 10.0$ y 20.0 partes por millón (ppm) y a partir de los datos obtenidos se construyó la recta de calibración, se obtuvieron la pendiente, intercepto, coeficiente de correlación (r) y de determinación ( $r 2$ ). En el gráfico 4 se muestra la recta de calibración en los tres días evaluados. Al revisar los datos obtenidos, los valores de $r$ y $r 2$ cumplieron el criterio de aceptación, adicionalmente la revisión visual demostró la correcta linealidad en el rango de trabajo seleccionado. Finalmente, en el análisis de varianza (ANOVA), el valor de $F$ calculado fue mayor al $F$ tabulado, con lo cual se demostró que existe relación lineal entre las variables. La tabla 4 muestra el análisis estadístico practicado con los datos obtenidos y el cumplimiento de los criterios de evaluación. Debido a que el intervalo de trabajo es aquel donde se demuestra la linealidad del método, estos mismos resultados son los empleados para este parámetro.

3. Exactitud (veracidad): para este parámetro se fortificaron nueve muestras de supositorios que contenían acetaminofén de una marca comercial, a tres diferentes concentraciones, cada uno por triplicado, dentro del rango de linealidad, utilizando una solución estándar de analito con una concentración conocida para tener concentraciones de fortificación de 1, 5 y 20 ppm. Al evaluar los resultados obtenidos los coeficientes de variación obtenidos para los tres niveles de fortificación fueron menores al $3 \%$ y los valores de t calculados fueron mucho más bajos que el $t$ tabulado. 


\section{Gráfico 3. Optimización de d-SPE}

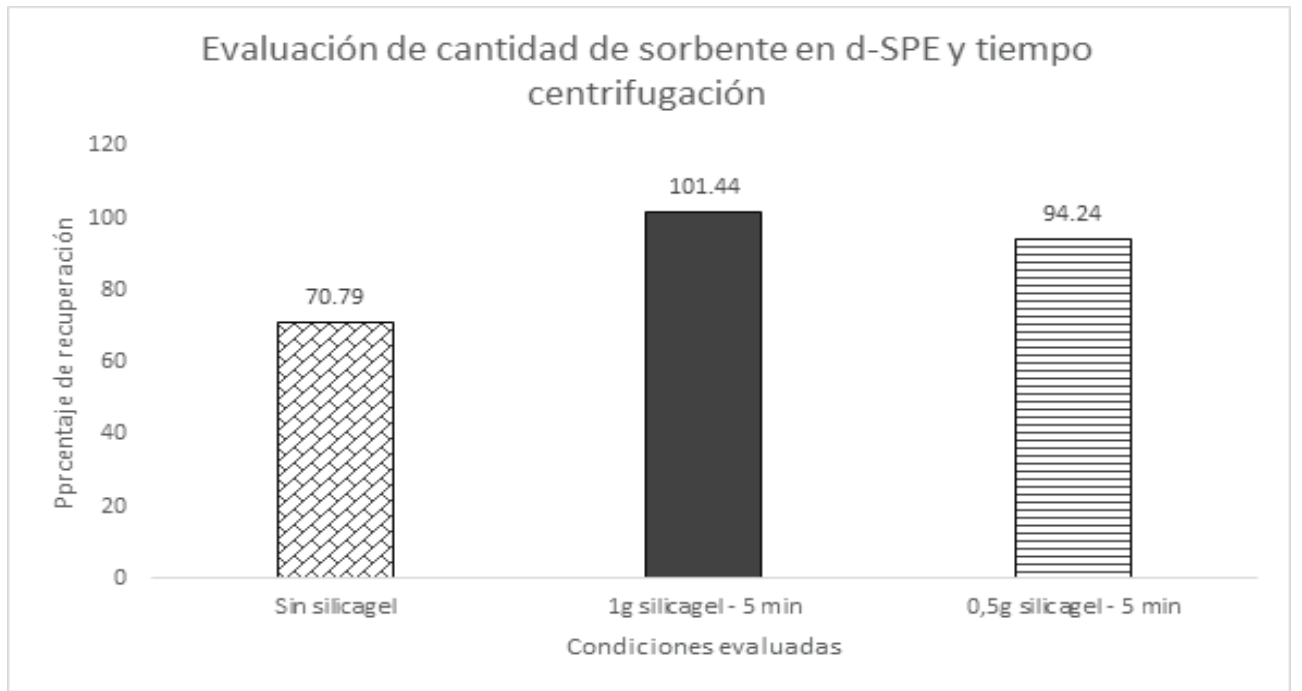

Fuente: Creación propia.

Gráfico 4. Curva de calibración obtenida durante la evaluación de la linealidad del método

Curva de calibración-Linealidad

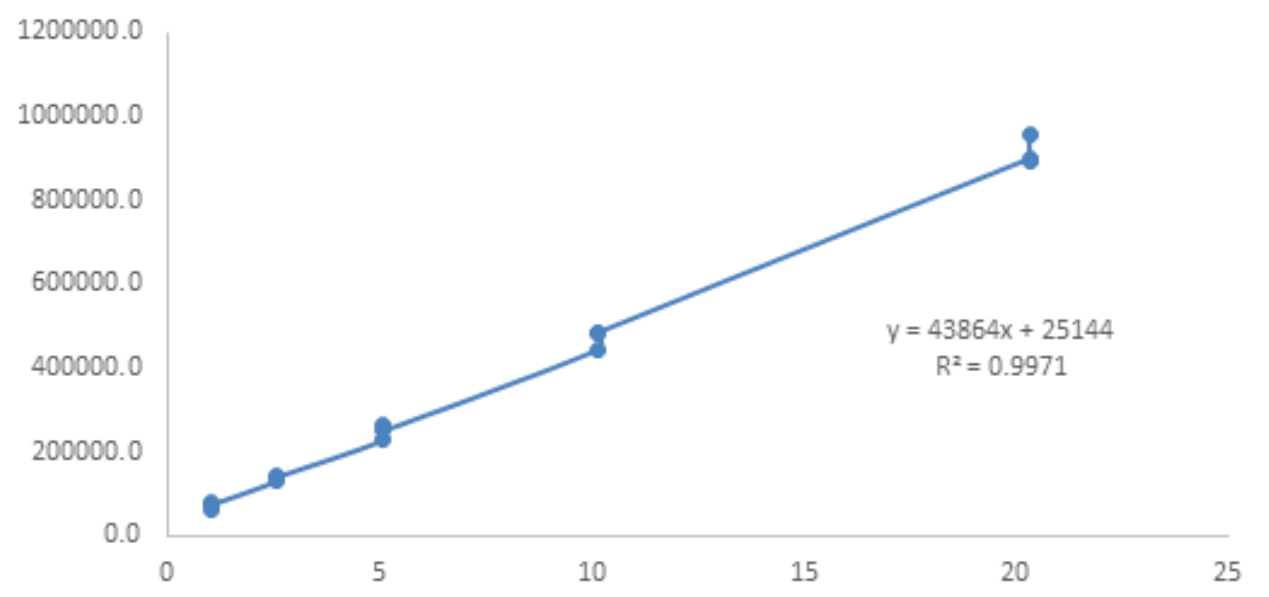

Fuente: Creación propia. 


\section{Figura 4. Esquema del procedimiento desarrollado}

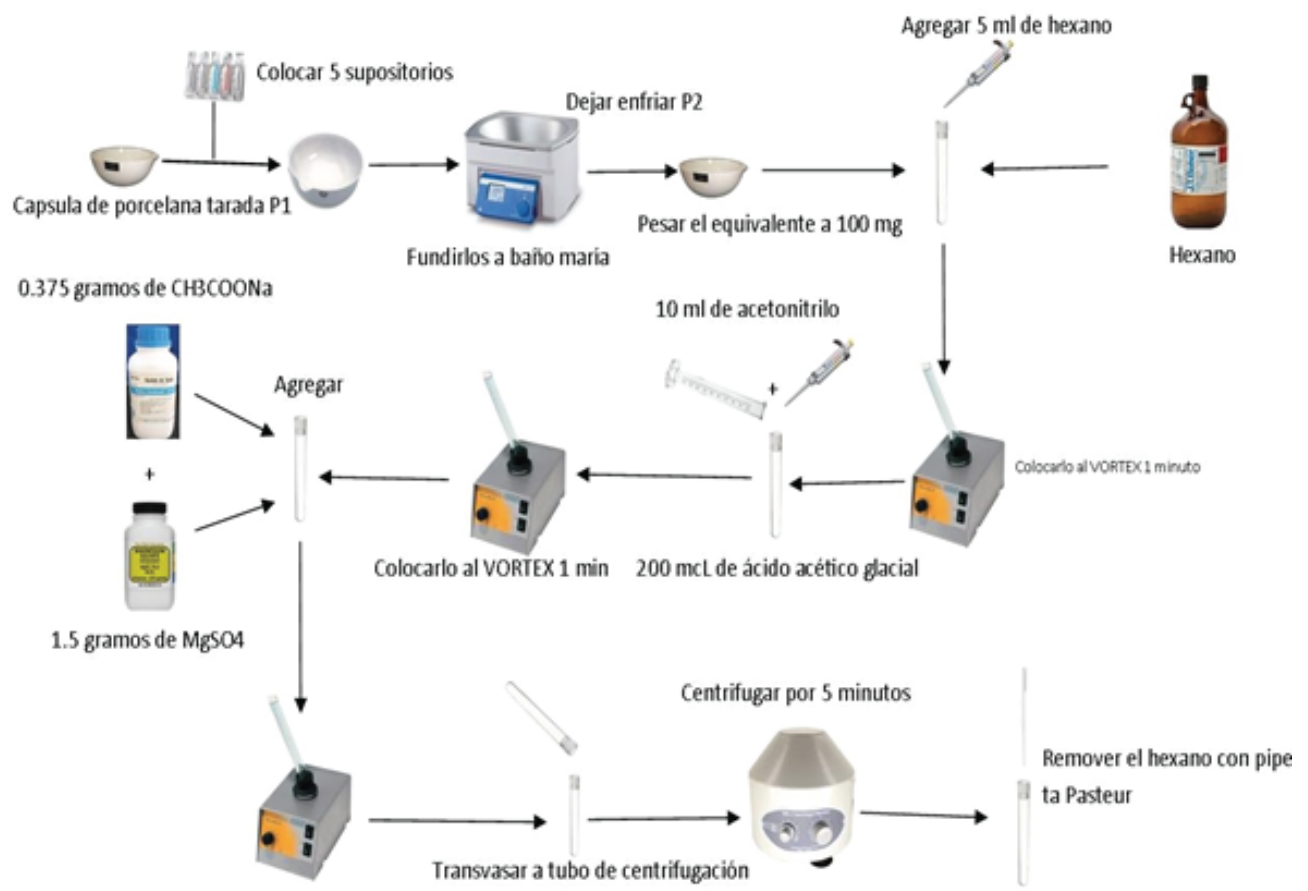

Colocarlo al VORTEX 1 minuto

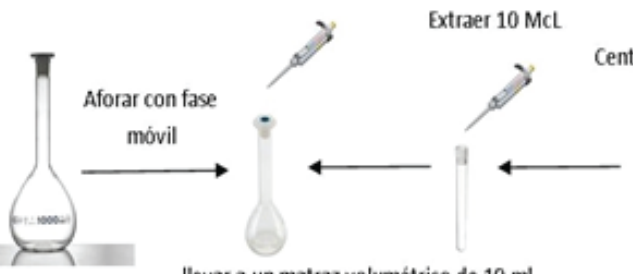

llevar a un matraz volumétrico de $10 \mathrm{ml}$

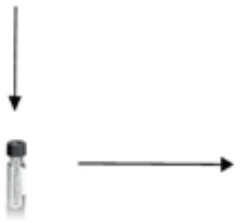

Inyectar la cantidad de $15 \mathrm{McL}$ al HPLC

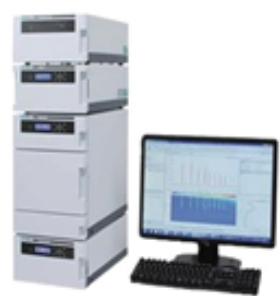


Tabla 4. Análisis de varianza para la linealidad del método

\begin{tabular}{|c|c|c|c|c|c|}
\hline Fuente & $\begin{array}{c}\text { Suma } \\
\text { cuadrados }\end{array}$ & $\begin{array}{l}\text { Grados de } \\
\text { libertad }\end{array}$ & $\begin{array}{c}\text { Cuadrados } \\
\text { medios del error }\end{array}$ & F calculado & F tabulado \\
\hline $\begin{array}{c}\text { Regresión } \\
\text { lineal }\end{array}$ & Explicada & 1 & $2.64654 \mathrm{E}+13$ & 84.8423544 & 0.39160113 \\
\hline Residual & Inexplicada & 13 & $3.11936 \mathrm{E}+11$ & & \\
\hline Total & Total & 14 & & & \\
\hline alfa & 0.05 & & & & \\
\hline
\end{tabular}

\begin{tabular}{|l|l|l|}
\hline \multicolumn{1}{|c|}{ Hipotesis de prueba } & Criterios de aceptación & \multicolumn{2}{c|}{ Resultado } \\
\hline Prueba para la regresión & Si F calculado es mayor que F & \\
\cline { 1 - 2 } Ho: concentración y señal no se relacionan lineal & tabulado se rechaza Ho y existe & Cumple \\
\cline { 1 - 1 } H1: concentración y señal se relacionan lineal & relación lineal entre las variables & \\
\hline
\end{tabular}

Fuente: Creación propia.

4.Precisión: se evaluó la repetibilidad y reproducibilidad intermedia del método. En el caso de la primera se analizaron seis muestras de supositorios a una concentración de 10 ppm, por el mismo analista en el mismo día y se obtuvo el coeficiente de variación, el cual resultó menor al $10 \%$. La precisión intermedia se evaluó para 2 analistas en diferentes días, con la determinación de seis muestras de supositorios de acetaminofén y calculando el coeficiente de variación de los resultados obtenidos. Asimismo, se practicó un análisis de varianza mediante un test $t$, cuyos resultados indicaron que no existen diferencias significativas en los resultados al cambiar el analista o el día del ensayo, puesto que el valor de $t$ calculado fue mucho menor que el valor tabulado, como se presenta en la tabla 5.

Tabla 5. Análisis de varianza para la reproducibilidad intermedia

\begin{tabular}{|c|c|c|c|c|c|}
\hline $\begin{array}{c}\text { Concentración } \\
(\mathrm{ppm})\end{array}$ & 10 & & & & \\
\hline $\begin{array}{l}\text { Promedio } \\
\text { Analista } 1\end{array}$ & $\begin{array}{c}\text { S } \\
\text { Analista } 1\end{array}$ & $\begin{array}{c}\text { Varianza } \\
\text { Analista } 1\end{array}$ & $\begin{array}{l}\text { Promedio } \\
\text { Analista } 2\end{array}$ & $\begin{array}{c}\text { S } \\
\text { Analista } 2\end{array}$ & $\begin{array}{c}\text { Varianza } \\
\text { Analista } 2\end{array}$ \\
\hline 83.97 & 7.65 & 58.65 & 106.61 & 11.84 & 140.28 \\
\hline $\begin{array}{c}\text { Varianza } \\
\text { estadística }\end{array}$ & $\begin{array}{c}\text { Grados de } \\
\text { libertad }\end{array}$ & $\mathrm{t}$ calculado & $\mathbf{t}$ tabulado & & \\
\hline 99.46 & 8 & -3.58 & 2.30 & & \\
\hline
\end{tabular}




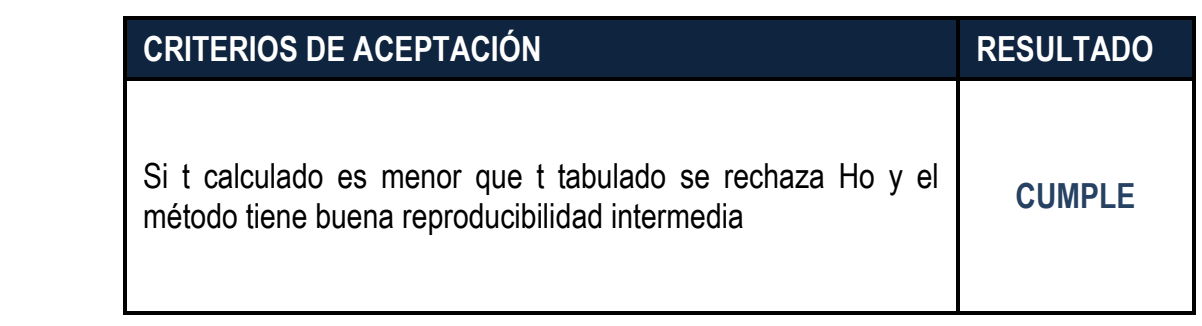

Fuente: Creación propia.

\section{CONCLUSIONES}

La aplicación de métodos analíticos baratos, rápidos, sencillos y seguros, es cada vez más necesario en el campo del análisis farmacéutico, por ello el desarrollo de técnicas de tratamiento de muestra que faciliten el aislamiento del o los analitos de interés previo a la determinación instrumental ha ido ganando mucho terreno en la química analítica. Con la presente investigación se logró desarrollar una variante del método QuEChERS, disminuyendo la cantidad de reactivos, el tiempo de extracción, lo mismo que con un procedimiento más sencillo al método de referencia que establece la farmacopea de los Estados Unidos XXXV para supositorios de acetaminofén.

Los diferentes parámetros del método de extracción fueron optimizados, incluyendo el solvente de dilución, solvente extractor, utilización de tampón, cantidad de sales empleadas, tiempo de centrifugación y empleó de sorbente en la etapa SPE-dispersiva, de modo que el porcentaje de recuperación en la extracción sea lo más elevado posible. Adicionalmente, se empleó el método desarrollado para determinar la cantidad de acetaminofén que presentan presentaciones comerciales de supositorios.

En el caso de la separación cromatográfica, se empleó la que establece la farmacopea, utilizando la separación en fase reversa con una fase móvil compuesta por agua y metanol en proporción de 3 a 1. El pico del analito se observó antes de los tres minutos, por lo que el tiempo total de análisis fue de tan solo cinco minutos.

Posteriormente al desarrollo, se validó el método alternativo, empleando las recomendaciones de la $\mathrm{ICH}$, evaluando los principales parámetros, dentro de los cuales se concluye que el método presentó una buena linealidad, así como una correcta especificidad. En cuanto a la exactitud se encontraron valores elevados de recuperación, muy cercanos al $100 \%$ de las concentraciones evaluadas, pese a que el procedimiento de extracción conlleva etapas en las que se pueden tener pérdidas del analito. 
Asimismo, los coeficientes de variación del método en la evaluación de la repetibilidad y reproducibilidad intermedia, no fueron mayores al $10 \%$ en la mayoría de los ensayos, debido a que las condiciones del laboratorio no se encuentran controladas, lo que sin duda alguna puede ser corregido en laboratorios que cuenten con una estructura propicia para disminuir estas variaciones. Por ello, se puede afirmar que el método presenta características que lo hacen aplicable por cualquier laboratorio dedicado al control de medicamentos.

La investigación demostró que el desarrollo de métodos alternos a los que presentan las normativas oficiales es posible, haciendo procedimientos con menor consumo de reactivos, tiempo y menor uso de solventes tóxicos. Con ello se puede afirmar que el método QuEChERS con las variaciones del caso, pudiese ser utilizado para matrices farmacéuticas laboriosas como supositorios, óvulos y similares, con lo cual se puede pensar que investigaciones en esta línea de investigación pueden seguirse efectuando.

\section{AGRADECIMIENTO}

A las autoridades de la Facultad por la oportunidad de utilizar el cromatógrafo líquido del laboratorio instrumental, además de aportar todos los reactivos e insumos necesarios durante la experimentación. Al Grupo de Investigación Análisis Químico, por compartir sus conocimientos y experiencias durante la investigación.

\section{BIBLIOGRAFÍA}

Anastassiades, M. L. (2003). Fast and Easy Multiresidue Method Employing Acetonitrile Extraction/Partitioning and "Dispersive Solid-Phase Extraction" for the Determination of Pesticide Residues in Produce. Journal of AOAC Inernational, 412-431.

Convención de la Farmacopea de los Estados Unidos de América. (2012). Farmacopea de los Estados Unidos de América. Rockville, MD: United States Pharmacopeial Convention.

Guideline, H. T. (2006). Validation of Analytical Procedures: Text and Methodology. International Conference on Harmonisation of technical Requirements for Registration of Pharmaceuticals for human use (pág. 1). Estados Unidos de Norteame- 
rica: $\mathrm{ICH}$.

Mitra, S. (2003). Chemistry, Sample preparation techniques in analytical. New Jersey: Wiley.

Moffat, A. C., Osselton, M. D., \& Widdop, B. (2005). Clarke's Analysis of Drugs and Poisons. Londres: Pharmaceutical Press.

Núñez, O., Gallart-Ayala, H., Martins, C., \& Lucci, P. (2012). New trends in fast liquid chromatography for food and environmental analysis. J. Chromatogr. A, 298-323.

Ponce, H. (27 de Junio de 2014). Desarrollo de un método analítico para determinada medios yodados de contraste de rayos $X$ en peces mediante LC-MS (HR). Trabajo de Fin de Máster. Tarragona, Tarragona, España: Universitat Rovira i Virgili.

Roberts II, L., \& Morrow, J. (2001). Analgésicos-Antipiréticos y antiinflamatorios, y fármacos antigotosos. En A. Goodman Gilman, J. Hardman, \& L. Limbird, Las bases farmacológicas de la terapéutica (págs. 697- 742). México, D.F.: McGrawHill.

RTCA. (2010). para la evaluaciónde la calidad de los productos farmacéuticos, Validación de métodos analíticos. Reglamento Técnico Centroamericano, (pág. 1). Tegucigalpa, M.D.C.

Snyder, L. R., Kirkland, J. J., \& Dolan, J. W. (2010). Introduction to modern Liquid Chromatography. Estados Unidos de Norteamerica: Wiley Sons.

Zhang, L., Liu, S., Cui, X., Pan, C., Zhang, A., \& Chen, F. (2012). A review of sample preparation methods for the pesticide residue analysis in foods. Cent. Eur. J. Chem., 900-. 